\title{
Oxidization Resistance in Vivo for Raspberry Flavone
}

\author{
Jinxu Sun ${ }^{1,2}$, Huixia Zhu ${ }^{1,2}$, Guangxiao Dong ${ }^{1^{*}}$ \\ ${ }^{1}$ Key Laboratory of Industrial Microbiology, Ministry of Education, \\ College of Biotechnology, Tianjin University of Science and Technology, Tianjin, China \\ ${ }^{2}$ Department of Biology, Hengshui College, Hengshui, China \\ Email: *bdsunjinxu@163.com
}

Received 2013

\begin{abstract}
The experiments on the effect of oxidization resistance in vivo for raspberry flavone extract manifests that the extract of raspberry flavone could effectively increase CAT, SOD and GSH-Px enzyme activity in rats' liver, kidney, blood and skeletal muscle and decrease decomposition product of lipid peroxidization, with a significant antioxidant effect.
\end{abstract}

Keywords: Raspberry; Flavone; Rats

\section{Introduction}

Flavonoid compound extensively exist in plants' fructification, stems, flowers and leaves, possessing a great many kinds of biological activities. Domestic and overseas scholars have studied and proved that flavonoid compounds are anti-neoplastic, antibacterial, anti free radical and anti-virus. In recent years, there are related reports on the studies on flavone oxidization resistance in plants, but few on raspberry flavone oxidization resistance $[1,2]$.

\section{Materials and Method}

\section{The first extract of raspberry flavone}

Weigh and take $100 \mathrm{~g}$ comminuted raspberry (first grade, bought from the pharmacy of Heng shui City Hospital of Chinese medicine. It was comminuted and sieved with 60 mesh and ready for use after drying at $50^{\circ} \mathrm{C}$ ) to put into triangular flask, and adding into $1 \mathrm{~L} \mathrm{95 \%}$ alcohol, which was conducted extract at $50^{\circ} \mathrm{C}$ with $36 \mathrm{~h}$ of extraction. During extraction, sonic extract was done at the $12^{\text {th }}$ and $24^{\text {th }}$ hour, with $300 \mathrm{~W}$ of ultrasonic power and $25 \mathrm{~min}$ of ultrasonic time. When the extract was at $50^{\circ} \mathrm{C}$ (Ultrasonoscope, JL-60DTH Shanghai Tianpu Analytical Instrument Co., Ltd.), jolt it constantly. After extraction, vacuum filtrate and vacuum freeze-dry to get the powder of crude extraction.

\section{The second extract of raspberry flavone}

The crude extraction liquid of rapberry flavone achieved in last step (rotated membrane evaporimeter, RE201D Shanghai Bocai Instrument Co., Ltd.) was vacuum rotated and evaporated to gain paste-like solids,

*Corresponding author. which were dissolved by ultrasound after adding into purified water. Extract for three times by petroleum ether, and the upper aqueous layer was taken to be extracted for three times by ethyl acetate, and yellow powder was got after vacuum rotation and evaporation.

\section{The purified products of raspberry flavone}

The powder of raspberry flavone extraction got in previous step was dissolved by $70 \%$ alcohol and purified by AB-8 rasin column (chemically pure Nanjing University Synthetic Resin Co., Ltd.), and then yellow powders were gained after vacuum rotation and evaporation.

The grouping and preparation of experimental rats

The rats for experiments (purchased from Chinese Academy of Medical Sciences Experimental Animal Institute): 3-week old male rats were used whose weights were between 80 and $100 \mathrm{~g}$.

The grouping and preparation of experimental rats: randomly divide rats into 5 groups with 7 rats in each group as high dose raspberry flavone group, low dose group, positive control group, negative control group and blank group. Force and feed blank group on normal saline and base without cholesterol every day; force feed negative control group on normal saline and cholesterol base every day; force and feed positive control group on $\mathrm{V}_{\mathrm{E}}$ and base with cholesterol every day, while the $\mathrm{V}_{\mathrm{E}}$ feeding amount was calculated according to $0.0025 \mathrm{~g} / \mathrm{kg}$ (body weight); feed high and low dose groups on fodder with cholesterol and high and low dose of raspberry flavone extraction every day. The raspberry flavone extraction was calculated based on $400 \mathrm{mg} / \mathrm{kg}$ and $100 \mathrm{mg} / \mathrm{kg}$ rat's weight; the addition amount of cholesterol in base was $0.5 \%$. After 6 weeks' feeding, rats were fast for $12 \mathrm{~h}$, and 7 rats were randomly taken to conduct experiments.

The fundamental ingredient of base (\%): starch: 50; 
soybean oil: 5.6; cellulose compound: 3.2; mineral element compound: 6.7 ; mixed vitamin: 1 ; protein: 23.2 ; water: 9.8 .

\section{The collection of rats' liver and kidney samples}

After 12 h' fasting, the experimental rats which were fed for 6 weeks were anaesthetized by 4\% pentobarbital sodium, and the dose was based on $40 \mathrm{mg} / \mathrm{kg}$ rats' weight. Take about 100 mg rats' skeletal muscle, renal cortex and liver, and $10 \mathrm{ml}$ blood. Add 0.9\% normal saline for $10 \mathrm{ml} / \mathrm{g}$. Grind tissues to get tissue homogenate, which was centrifuged at $4^{\circ} \mathrm{C}, 4000 \mathrm{rpm}$ for $15 \mathrm{~min}$, and take the supernatant to detect CAT, SOD and GSH-Px enzyme activity, and detect MDA [3].

\section{The determination of CAT activity}

CAT can resolve $\mathrm{H}_{2} \mathrm{O}_{2}$, and this reaction can be rapidly terminated by adding ammonium molybdate. The extra $\mathrm{H}_{2} \mathrm{O}_{2}$, together with ammonium, can form a kind of faint yellow complex compound, and its quantity of generation can be detected at $405 \mathrm{~nm}$, so as to calculate CAT activity. CAT kit used in experiments to detect was A007-1 (Nanjing Jiancheng Bioengineering Institute). 1 umol $\mathrm{H}_{2} \mathrm{O}_{2}$ which was resolved by $1 \mathrm{mg}$ tissue protein was taken as a unit of activity [4], shown by U/ml.

\section{The determination of SOD activity}

The determination of SOD activity with the method of xanthine oxidase Xanthine and xanthine oxidase reaction system can produce superoxide anion free radical, which oxidized hydroxylamine to form nitrite that demonstrated to be purple when affected by chromogenic agent. The absorbance was detected at $550 \mathrm{~nm}$ to calculate SOD activity which was shown by U/mg protein. SOD kit used in experiments was A001-1 (Nanjing Jiancheng Bioengineering Institute).

\section{The determination of GSH-Px activity}

Glutathione peroxidase can promote $\mathrm{H}_{2} \mathrm{O}_{2}$ to react with reduced type glutathione for generating oxidized glutathione and water. The activity of glutathione peroxidase can be shown by enzymatic reaction velocity. By measuring the consumption of reduced type glutathione in enzymatic reaction, enzyme activity can be obtained. In reaction system, glutathione concentration decreased by $1 \mathrm{umol} / \mathrm{L}$, which was an activity unit. In the experiment,
GSH-Px kit A005-1 was employed to determine [5] (Nanjing Jiancheng Bioengineering Institute).

The determination of MDA content

The determination was by means of TBA (Thiobarbituric Acid Test). MDA kit used in the experiments was A003-1 (Nanjing Jiancheng Bioengineering Institute).

The determination of total protein content in tissues

By means of Coomassie brilliant blue protein determination kit A045-2 (Nanjing Jiancheng Bioengineering Institute), the determination was conducted and shown by $\mathrm{mg} / \mathrm{mL}$.

The statistical analysis

SPSS18.0 was used in statistical analysis, and the data was $\mathrm{x} \pm \mathrm{SD}, \mathrm{a}=0.05$.

\section{Results and Conclusion}

According to the analysis of oxidization resistance activity data in vitro of raspberry flavone extraction, among raspberry flavone crude extraction, raspberry flavone extract and raspberry flavone purified matters, raspberry flavone purified matters group has a greater ability in scavenging hydroxyl, $\mathrm{H}_{2} \mathrm{O}_{2}$ and DPPH than the other two ones. Therefore, raspberry flavone purified matters was chosen to be the raw material of rats' in vivo oxidization resistance experiments to conduct analysis, while the results are as follows:

The results of rats' liver oxidization resistance activity

From Table 1, it can be seen that in rats' liver tissues, high dose group has the highest CAT, SOD and GSH-Px activity (cholesterol and high dose raspberry flavone purified matters were fed), being significantly different from other four groups ( $\mathrm{p}<0.05$ ); the second highest one is low dose group (low dose raspberry flavone purified matters and cholesterol were fed), which differs from some groups remarkably. Between blank, positive and negative groups, there is also some significance of difference, which is small. The high dose purified matters group has an obvious higher MDA content than other groups, being significantly different from other groups (p

Table 1. The determination result for big mouse liver CAT, SOD, GSH-Px, MDA.

\begin{tabular}{|c|c|c|c|c|}
\hline $\begin{array}{ll}\text { Treatment } & \text { Item } \\
\end{array}$ & CAT (U/mL) & SOD (U/m) & GSH-Px & MDA (mol/mg) \\
\hline The Blank control & $45.57 \pm 1.16$ & $204.06 \pm 1.47$ & $626.41 \pm 5.34$ & $9.89 \pm 0.43$ \\
\hline The Negative control & $53.58 \pm 1.60^{\Delta}$ & $212.25 \pm 1.63^{\Delta}$ & $638.70 \pm 3.54^{\Delta}$ & $12.96 \pm 0.39^{\Delta}$ \\
\hline The Positive control & $59.09 \pm 1.77^{\Delta \#}$ & $219.06 \pm 1.14^{\Delta \#}$ & $647.81 \pm 3.85^{\Delta \#}$ & $7.23 \pm 0.29^{\Delta \#}$ \\
\hline The low dose group & $61.01 \pm 1.55^{\Delta \#}$ & $229.92 \pm 2.35^{\Delta \#}$ & $698.09 \pm 4.76^{\Delta \#}$ & $6.63 \pm 0.08^{\Delta \#}$ \\
\hline The high dose group & $69.19 \pm 1.38^{\Delta \# \text { 访 }}$ & $243.75 \pm 1.69^{\Delta \# \text { 它 }}$ & 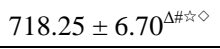 & $4.21 \pm 0.13^{\Delta \# c^{\# \diamond}}$ \\
\hline
\end{tabular}

Note: “ $\Delta$ ” stands for the significant difference when compared with blank control; “\#” stands for the significant difference compared with negative control; “ “㧧” stands for the significant difference compared with purified matters of low dose; “»” stands for the significant difference compared with purified matters of low dose. 
$<0.05$ ), which demonstrates that high dose raspberry flavone purified matters can increase the enzymes of oxidization resistance and scavenging free radical in rats' liver, benefiting removing the free radical in mice's livers, increasing liver's oxidization resistance, which is effective in oxidization resistance.

The result of detecting rats' kidney oxidization resistance activity

Table 2 shows that, being similar to rats' liver experiments, high dose group has the highest CAT, SOD and GSH-Px activity in rats' kidney, being significantly different from other four groups ( $\mathrm{p}<0.05)$. The second one is low dose group (low dose raspberry flavone purified matters and cholesterol were fed), which differs from some groups remarkably. The high dose purified matters group has a obvious lower MDA content than other groups, being significantly different from other groups ( $\mathrm{p}$ $<0.05$ ), which demonstrates that high dose raspberry flavone purified matters can increase the enzymes of oxidization resistance and scavenging free radical in rats' kidney, being propitious to remove the free radical in mice's kidneys, increasing kidney's oxidization resistance, which is effective in oxidization resistance. This experi- mental result is consistent with the oxidization resistance of rats' liver.

The result of detecting rats' blood resistance activity

From the results of Table 3, CAT, SOD and GSH-Px activity in rats' blood, there is difference in the groups. By comparison, the purified matters of high dose have an obvious higher activity than other groups, being obviously different from others $(\mathrm{p}<0.05)$. Between purified matters of low dose and other groups, there is some significant difference, while the difference is not significant between the remaining groups. It demonstrates that after feeding, raspberry flavone purified matters can markedly increase the activity of free radical scavenging enzyme in rats' blood and strengthen the oxidization resistance of rats' blood. MDA, the decomposition product of lipid peroxide, is higher in high dose purified mattes group than in other groups, and there is significant difference, which shows that high

The result of detecting rats' skeleton muscular tissue resistance activity

The result of Table 4 demonstrates to be consistent with the experimental results above. CAT, SOD, GSH-

Table 2. The determination result for big mouse kidney CAT, SOD, GSH-Px, MDA.

\begin{tabular}{|c|c|c|c|c|}
\hline Treatment Item & CAT (U/mL) & SOD (U/m) & GSH-Px & MDA (mol/mg) \\
\hline The Blank control & $84.79 \pm 0.93$ & $203.47 \pm 2.99$ & $409.40 \pm 3.78$ & $18.47 \pm 0.86$ \\
\hline The Negative control & $91.41 \pm 1.26^{\Delta}$ & $214.30 \pm 5.39^{\Delta}$ & $423.44 \pm 1.89^{\Delta}$ & $19.66 \pm 0.94^{\Delta}$ \\
\hline The Positive control & $97.36 \pm 1.96^{\Delta \#}$ & $209.71 \pm 2.40$ & $440.64 \pm 2.19^{\Delta \#}$ & $15.70 \pm 0.56^{\Delta \#}$ \\
\hline The low dose group & $105.59 \pm 2.24^{\Delta \text { \#江 }}$ & $227.02 \pm 5.17^{\Delta \# \text { \#访 }}$ & $453.67 \pm 2.65^{\Delta \# \text { 永 }}$ & $13.20 \pm 0.55^{\Delta \# \text { 纷 }}$ \\
\hline The high dose group & $121.12 \pm 1.45^{\Delta \# \text { 访〉 }}$ & $248.10 \pm 4.47^{\Delta \# \text { 永 } \diamond}$ & $469.40 \pm 1.05^{\Delta \# \text { 访 } \diamond}$ & $9.91 \pm 0.65^{\Delta \# \text { 的 }}$ \\
\hline
\end{tabular}

Note: “ $\Delta$ ” stands for significant difference when compared with blank control; “\#” stands for the significant difference compared with negative control; “柿”, stands for the significant difference compared with positive control group; “»”, stands for the significant difference compared with purified matters of low dose.

Table 3. The determination result for big mouse blood CAT, SOD, GSH-Px, MDA.

\begin{tabular}{|c|c|c|c|c|}
\hline Treatment Item & CAT (U/mL) & $\mathrm{SOD}(\mathrm{U} / \mathrm{m})$ & GSH-Px & MDA (mol/mg) \\
\hline The Blank control & $76.12 \pm 2.61$ & $187.39 \pm 5.38$ & $391.67 \pm 2.53$ & $18.48 \pm 0.58$ \\
\hline The Negative control & $84.47 \pm 2.50^{\Delta}$ & $200.41 \pm 2.56^{\Delta}$ & $417.06 \pm 4.56^{\Delta}$ & $19.17 \pm 0.39$ \\
\hline The Positive control & $92.20 \pm 1.37^{\Delta \#}$ & $208.71 \pm 11.47^{\Delta}$ & $426.67 \pm 6.09^{\Delta}$ & $14.55 \pm 0.63^{\Delta \#}$ \\
\hline The low dose group & $94.89 \pm 0.79^{\Delta \# \text { 约 }}$ & $209.29 \pm 1.03^{\Delta}$ & $441.38 \pm 5.71^{\text {倣 }}$ & $13.12 \pm 0.96^{\Delta \#}$ \\
\hline The high dose group & $109.55 \pm 1.18^{\Delta \# \text { 访〉 }}$ & 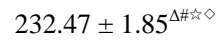 & 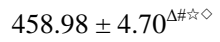 & $10.86 \pm 0.34^{\Delta \# \text { 访 }}$ \\
\hline
\end{tabular}

Note: “ $₫$ ” stands for significant difference when compared with blank control; “\#” stands for the significant difference compared with negative control; “ “柿” stands for the significant difference compared with positive control group; “»”, stands for the significant difference compared with purified matters of low dose.

Table 4. The CAT, SOD, GSH-Px, MDA determination result for big mouse skeleton muscular tissue.

\begin{tabular}{|c|c|c|c|c|}
\hline Treatment & CAT (U/mL) & $\mathrm{SOD}(\mathrm{U} / \mathrm{m})$ & GSH-Px & MDA (mol/mg) \\
\hline The Blank control & $84.04 \pm 2.40$ & $193.66 \pm 4.30$ & $303.85 \pm 6.21$ & $16.80 \pm 0.63$ \\
\hline The Negative control & $90.51 \pm 0.90^{\Delta}$ & $197.51 \pm 1.86$ & $288.01 \pm 9.19^{\Delta}$ & $17.81 \pm 0.46$ \\
\hline The Positive control & $95.43 \pm 1.65^{\Delta}$ & $210.17 \pm 2.85^{\Delta \#}$ & $320.06 \pm 1.54^{\Delta \#}$ & $15.40 \pm 0.57^{\#}$ \\
\hline The low dose group & 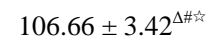 & $225.77 \pm 4.40^{\Delta \# \text { 坊 }}$ & $330.94 \pm 3.43^{\Delta \#}$ & $12.24 \pm 0.26^{\Delta \# \text { 仯 }}$ \\
\hline The high dose group & $122.34 \pm 1.20^{\Delta \# \text { 放 }}$ & $239.74 \pm 1.99^{\Delta \# \text { 弥 }}$ & $350.62 \pm 2.01^{\Delta \# \text { 弥 }}$ & $10.17 \pm 0.48^{\Delta \# \text { 纺 }}$ \\
\hline
\end{tabular}

Note: “₫” stands for significant difference when compared with blank control; “\#” stands for the significant difference compared with negative control; “ “㕫” stands for the significant difference compared with positive control group; “»»" stands for the significant difference compared with purified matters of low dose. 
Px activity in rats' musculus skeleti are significantly higher in high dose raspberry flavone purified matters than in other groups, while MDA is lower, being significantly different from other groups, followed by low dose raspeberry flavone purified matters. It shows that raspberry flavone purified matters can obviously increase the activity of free radical scavenging enzyme in rats' musculus skeleti, strengthening the oxidization resistance of rats' blood, decreasing lipid peroxidization and improving oxidization resistance.

\section{DISCUSSION}

1) In recent years, the relation of free radical and many diseases has been increasingly aroused great attention. The development of free radical biomedicine enables the natural antioxidant, high efficient and low-toxic free radical scavenger to be the research hotspot of biological chemistry and medicine and pharmacology [6]. Oxidization resistance is considered to be the most important mechanism of flavonoid compound. The flavonoid compound is well received in western countries, whose oxidization resistance and other biological activities are also gradually paid attention to by Chinese.

2) Dried fruit of raspberry is used as both food and medicine, being effective in protecting liver and kidney and preventing and resisting cancers [7]. One of the major functional matters in raspberry is flavonoid compound. The identified raspberry flavonoid compounds are mainly kaempferol, quercetin and tiliroside, which are special in structure and effective with more than $90 \%$ of bioavailability when compared with most flavones.

\section{REFERENCES}

[1] J. Y. Cha and Y. S. Cho, "Effect of Hesperetin, a Citrus Flavonoid, on the Liver Triacylglycerol Content and Pho- sphatidate Phosphohydrolase Activity in Orotic Acid-Fed Cha J. Y, Rats,” Plant Foods for Human Nutrition, Vol. 56, 2001, pp. 349-358. http://dx.doi.org/10.1023/A:1011884200848

[2] H. Koyuncu, B. Beerkarda and F. Baykut, "Preventive Effect of Hesperidin against Inflammation in Mouse Skin Caused by Tumor Promoter," Anticancer Research, Vol. 19, 2008, pp. 3237-3241.

[3] K. Kawaguchi, S. Kikuchi, R. Hasunuma, et al., "Suppression of Infection-Induced Endotoxin Shock in Mice by a Citrus Flavanone Naringin,” Planta Medica, Vol. 70, 2004, pp. 17-22. http://dx.doi.org/10.1055/s-2004-815449

[4] E. A. Kurowska and J. A. Manthey, "Hypolipidemic Effects and Absorption of Citrus Polymethoxylated Flavones in Hamsters with Diet-Induced Hypercholesterolemia," Journal of Agricultural and Food Chemistry, Vol. 52, 2007, pp. 2879-2886. http://dx.doi.org/10.1021/jf035354z

[5] Q. F. Zhang and Z. R. Zhang, “Antioxidant Activity of Rhizoma Smilacis Glabrae Extracts and Its Key Constituent Astilbin,” Food Chemistry, Vol. 115, 2008, pp. 297303. http://dx.doi.org/10.1016/j.foodchem.2008.11.053

[6] A. Ardestani and R. Yazdanpast, "Antioxidant and Free Radical Scavenging Potential of Achillea Santolina Extracts," Food Chemistry, Vol. 104, 2007, pp. 21-29. http://dx.doi.org/10.1016/j.foodchem.2006.10.066

[7] L. R. Della, R. Loggia and S. Sosa, "Anti-Inflammatory Activity of Ginkgobiloba Flavonoids,” Planta Medicine, Vol. 59, 2006, p. A588. 